\title{
Fast Extraction of Somatosensory Evoked Potential using RLS Adaptive Filter Algorithms
}

\author{
Zhaoli REN, Yuexian ZOU \\ Advanced Digital Signal Processing Lab \\ Shenzhen Graduate School of Peking University \\ Shenzhen, Guangdong, China
}

\author{
Zhiguo ZHANG, Yong HU \\ Department of Orthopaedics \& Traumatology \\ The University of Hong Kong \\ Hong Kong SAR, China
}

\begin{abstract}
This paper evaluates the efficacy of the recursive least squares (RLS) in adaptive noise canceller (RLS-ANC) for fast extraction of somatosensory evoked potentials (SEPs). The RLSANC method was verified by simulation of electroencephalography (EEG) and Gaussian noise contaminated SEP signals at different signal-to-noise ratios (SNRs). RLS was found to converge faster than the least mean squares (LMS) algorithm in ANC, i.e. SEP extraction by RLS-ANC required fewer trials than LMS-ANC. Experimental results showed that RLS-ANC with less than 50 trials could provide similar performance in SEP extraction to those extracted by the conventional ensemble averaging with 500 trials even at SNR of 20dB.
\end{abstract}

Keywords-SEP; Adaptive Filter; RLS-ANC; LMS-ANC

\section{INTRODUCTION}

Somatosensory Evoked Potentials (SEPs) are brain electrical physiological signals elicited by the direct electrical stimulation of peripheral nerves [1]. SEP has been widely used during the clinical testing and monitoring of the spinal cord and the central nervous system. However, a severe problem in practice is that the SEP signals recorded in operating theaters are always contaminated by both electrical and biological noises in nature. Hence the noninvasive measurement of the SEP suffers from very poor signal-to-noise ratio (SNR), which is typically less than $0 \mathrm{~dB}$ and less than $-20 \mathrm{~dB}$ in the presence of larger noise interference [2].

Ensemble Averaging (EA) is the most commonly-used technique to extract the measurable and reliable SEP signals [3]. However, it needs a huge number of the SEP samples and a long period of time. Moreover, SEP is not a deterministic stationary signal, therefore, the response waveform of each stimulus may be different from each other. This variation property of the SEP cannot be reflected after averaging of thousands of trials [4]. Therefore, a more effective and reliable technique is expected to minimize the number of trials of EA and to extract SEP dynamically and rapidly.

Recently, adaptive filtering emerged as a reliable and efficient technique for the extraction of the evoked potential [5], and the least mean squares (LMS) based adaptive noise canceller (ANC) was found to be a fast, simple, and reliable SEP extraction method for intraoperative spinal cord monitoring [5]. The LMS algorithm is famous for its simplicity in concept and implementation, but it has a relatively slow convergence rate and is sensitive to the noise disturbance, which is remarkable in SEP monitoring. Therefore, we try to

This work is supported by Shenzhen Science and Technology Program (No. 08CXY-01)

Contact Author: zouyx@szpku.edu.cn employ other adaptive filtering algorithms in ANC for SEP extraction instead of the conventional LMS algorithm.

Recursive least squares (RLS) is a stable and accurate adaptive filtering algorithm because it updates the estimate using all the past available information, instead of the instantaneous measurement and error values in LMS. In principle, compared with LMS, RLS exhibits faster convergence rate and is less sensitive to noise disturbance, but at expense of a heavier computational load [6]. Although the RLS algorithm has been employed in a number of ANC applications, its application to the extraction of evoked potentials is very limited and the comparison between RLSbased and other adaptive filtering algorithms based ANC methods is never conducted.

In this paper, we propose the adoption of the RLS algorithm in the ANC method and applying the RLS-ANC method to extract simulated and recorded SEP signals. The performance of the RLS-ANC, LMS-ANC, and EA methods regarding to SEP extraction are evaluated and compared quantitatively.

\section{MATERIALS AND MetHOdS}

A. RLS-ANC

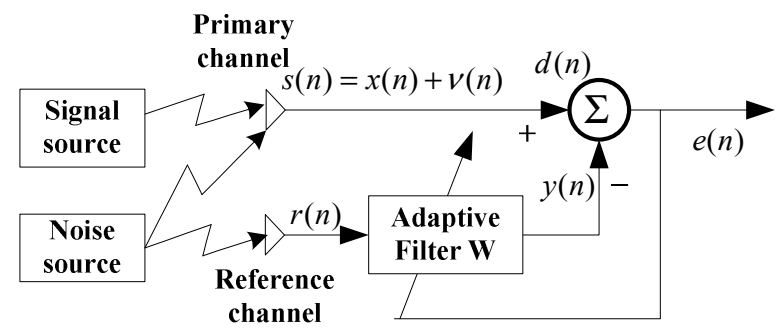

Figure 1. A block diagram of adaptive noise canceller (ANC) with one reference channel

RLS-ANC in this paper means the ANC filter using RLS algorithm. In Figure 1., a block diagram of ANC [7] is illustrated. The primary channel receives the signal $\mathrm{x}(\mathrm{n})$ plus an uncorrelated noise $\mathrm{v}(\mathrm{n})$. The reference channel receives a noise $\mathrm{r}(\mathrm{n})$ which is uncorrelated with the signal $\mathrm{x}(\mathrm{n})$, but correlated in some unknown way with the noise $\mathrm{v}(\mathrm{n})$. In Fig 1, s(n) represents the raw SEP recordings, which is composed of the SEP signal $x(n)$ and the noise $v(n)$. The reference channel $r(n)$ represents a noise source correlated with the noise $v(n)$. The noise $\mathrm{r}(\mathrm{n})$ is filtered to produce an output which is a close 
replica of $\mathrm{v}(\mathrm{n})$. This output is subtracted from the primary channel to produce the system output. The basic objective of the adaptive filter is to set its weight vector in such a way that its output tries to minimize a meaningful objective function involving the reference channel signal.

The recursive formulas of RLS algorithm operate in seven major steps:

Step1. Initialization

$$
\boldsymbol{W}_{M}(-1)=0, \boldsymbol{P}_{M}(n-1)=\delta^{-1} \boldsymbol{I}_{M}
$$

where $\mathrm{M}$ is the filter order of the RLS-ANC, $\delta$ can be the inverse of an estimation of the input signal power.

Step2. Calculation of the adaptive filter output

$$
y(n)=\boldsymbol{r}_{M}^{T}(n) \boldsymbol{W}_{M}(n-1)
$$

Step3. Estimation of the error

$$
e_{M}(n)=d(n)-y(n)
$$

Step4. Calculation of the Kalman gain vector

$$
\boldsymbol{K}_{M}(n)=\frac{\boldsymbol{P}_{M}(n-1) \boldsymbol{r}_{M}^{*}(n)}{\lambda+\boldsymbol{r}_{M}^{T}(n) \boldsymbol{P}_{M}(n-1) \boldsymbol{r}_{M}^{*}(n)}
$$

where $^{\lambda}$ is the forget factor.

Step5. Update of the inverse correlation matrix

$$
\boldsymbol{P}_{M}(n)=\frac{1}{\lambda}\left[\boldsymbol{P}_{M}(n-1)-\boldsymbol{K}_{M}(n) \boldsymbol{r}_{M}^{T}(n) \boldsymbol{P}_{M}(n-1)\right]
$$

Step6. Calculation of the filter weights

$$
\boldsymbol{W}_{M}(n)=\boldsymbol{W}_{M}(n-1)+\boldsymbol{K}_{M}(n) e_{M}(n)
$$

Step7. Go to the next instant, $n=n+1$

Since $\boldsymbol{K}_{M}(n)$ is a square matrix changed with $\mathrm{n}$, as a measure of the autocorrelation matrix, it indicates that, at different time, every element of $\boldsymbol{W}_{M}$ is adjusted with the new input data at a different weight, which explains why RLS has a fast convergence [8].

\section{B. Simulation Signals}

To compare the performances of ANC and EA, a simulation study was conducted. The SEP signals were collected over $\mathrm{Cz}^{\prime}$ ( $2 \mathrm{~cm}$ posterior to $\mathrm{Cz}, 10-20$ international system for EEG electrode placement) versus the Fz of the 1020 system. The stimulation for SEP recording was applied on the posterior tibial nerve with the duration of $0.3 \mathrm{~ms}$, the rate of $5.1 \mathrm{~Hz}$ and the constant current of 10 to $30 \mathrm{~mA}$. The signals were amplified one hundred thousand times, band-pass filtered at $20-3000 \mathrm{~Hz}$. All the SEP signals were acquired and recorded to a computer with 12-bit resolution and the sampling rate of 5 $\mathrm{kHz}$. We collected 500 trials for one subject and then the average of these trials is taken as a standard template for the simulation. Figure 2. shows the SEP template.

The reference noise was simulated by continuous EEG plus white Gaussian noise (WGN). Continuous EEG is the major source of noise found in the SEP recordings. The simulated EEG was recorded from the awaken subjects at a sitting position in a quiet environment. White Gaussian noise is a kind of noise having a frequency spectrum continuous and uniform over a specified frequency band. EEG signals were collected over $\mathrm{Cz}$ and $\mathrm{A} 1$ (auricular) versus $\mathrm{Fz}$, respectively. The $\mathrm{Cz}-\mathrm{Fz}$ EEG plus WGN was superimposed onto the SEP to form the noise-contaminated SEP. A1-Fz EEG was used for the simulation of input to reference signals of theadaptive filter. Figure 3. shows the simulated SEP signal at $-15 \mathrm{~dB}$ with EEG and WGN.

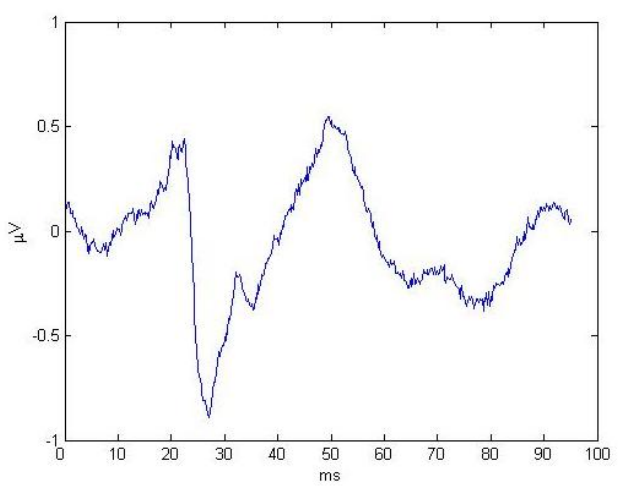

Figure 2. A SEP template obtained from ensemble averaging of 500 trials.

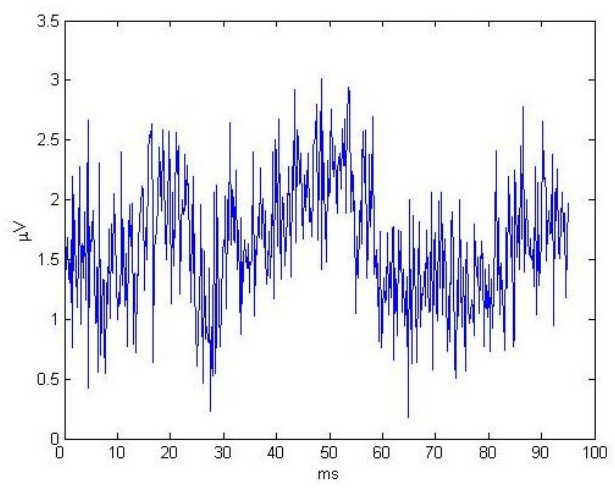

Figure 3. Simulated SEP signal at $-15 \mathrm{~dB}$ with $\mathrm{EEG}$ and WGN

\section{Signal Processing and Data Analysis}

In this study, the step size $\mu$ and the filter order $\mathrm{N}$ of the LMS were chosen as 0.000002 and 10 , respectively, while the forgetting factor $\lambda$ and the filter order of the RLS were chosen as 0.99 and 10, respectively. All the processing programs were implemented in MATLAB 7.0.

To quantitatively evaluate the performance of three different algorithms, three measures were employed: signal distortion index, waveform correlated coefficients, latency and peak-to-peak amplitude differences. Due to page limitation, the details of three measures can be found in [5].

\section{EXPERIMENTAL RESULTS}

Figure 4. gives the SEP plots for EA, LMS-ANC, and RLS-ANC algorithms. 
From Figure 4. , it's apparent that the RLS-ANC algorithm could extract the most similar signal with the template. Experimental results also showed that the RLS-ANC needs about 50 trials for convergence at $-10 \mathrm{~dB}$.

Using signal distortion index as a guideline, the RLS-SEP displayed the best results. By 10 trials at $-10 \mathrm{~dB}$, the RLS-ANC gives a SDI below 0.1 (Figure 5. a). On the contrary, the EASEP didn't reach the 0.1 after 50 trials and the LMS-SEP just reached the 0.2 after 25 trials. When SNR was chosen as -20 $\mathrm{dB}$, the difference was more obvious. After 50 trials, the SDI of the RLS-SEP was lower than 0.2 while the SDI of EA and the LMS-SEP reached almost the 0.6 and 0.4 , respectively (see Figure 5. ).

Among 5 simulated cases, the mean correlated coefficients between the EA-SEP waveform, LMS-SEP waveform, RLSSEP waveform and the template waveform are shown in Table 1. For all the SNR levels, the correlation coefficients between RLS-SEP and the template were always the highest among three SEP signals. The correlations between the SEPs and the template decreased while the input SNR decreased. At $-10 \mathrm{~dB}$ SNR, the correlation coefficients were almost at 0.9 , which had small differences in the EA-SEP, LMS-SEP, and RLS-SEP waveforms than those in lower SNR levels.

The latency and the amplitude are two important clinical parameters in the nervous system examination and the intraoperative spinal cord monitoring. Their percentage differences are presented in Table 2. According the data in the table, RLS-SEP didn't show a significant difference from the template.

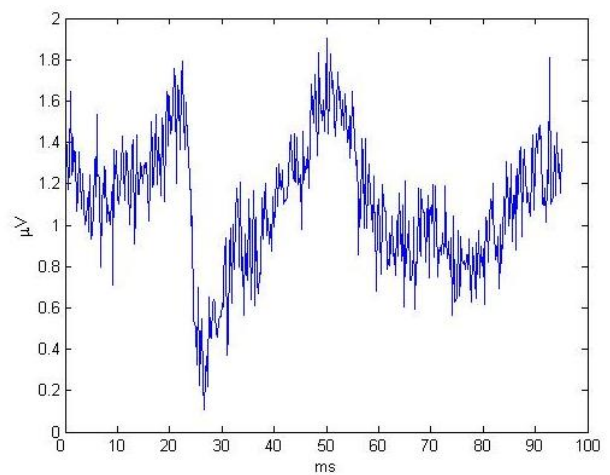

(a) 50 trials EA-SEP waveform

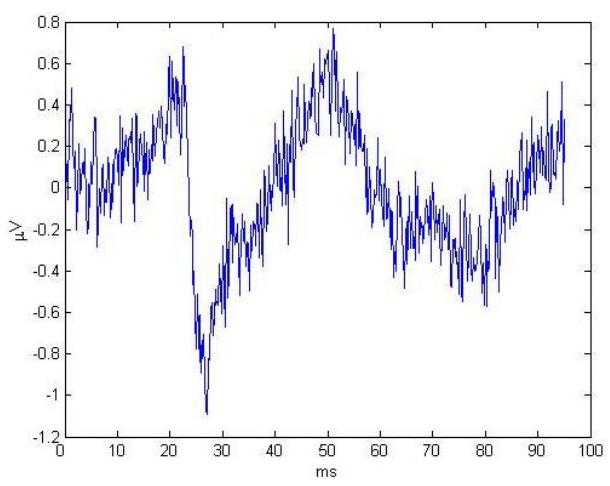

(b) 50 trials LMS-SEP waveform

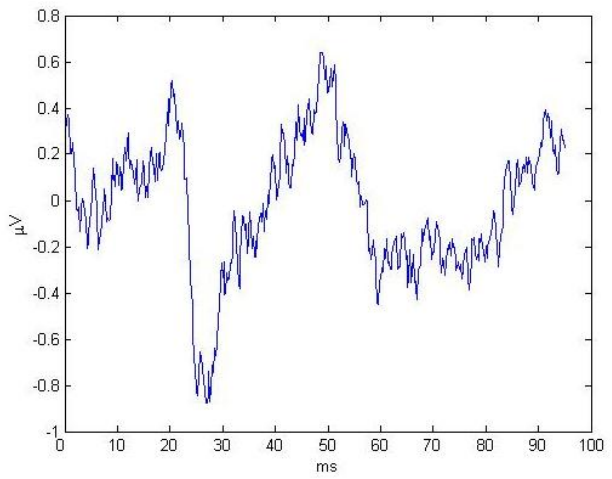

(c) 50 trials RLS-SEP waveform

Figure 4. SEP waveforms by 50 trials at $-15 \mathrm{~dB}$ : (a) EA-SEP; (b) LMS-SEP; (c) RLS -SEP

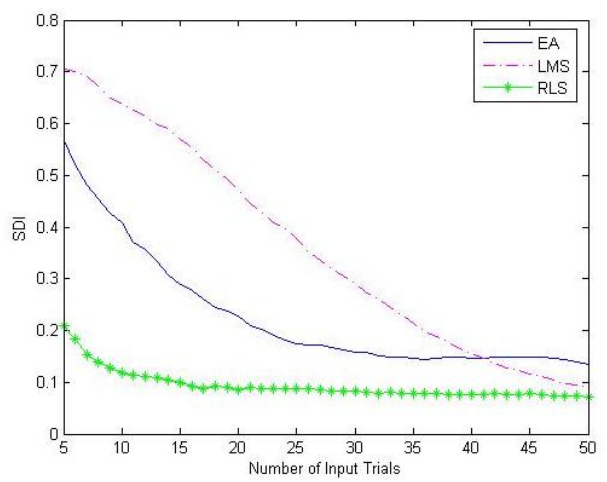

(a) Comparison of EA-SEP, LMS-SEP and RLS-SEP with template distortion index against trial number $(-10 \mathrm{~dB})$

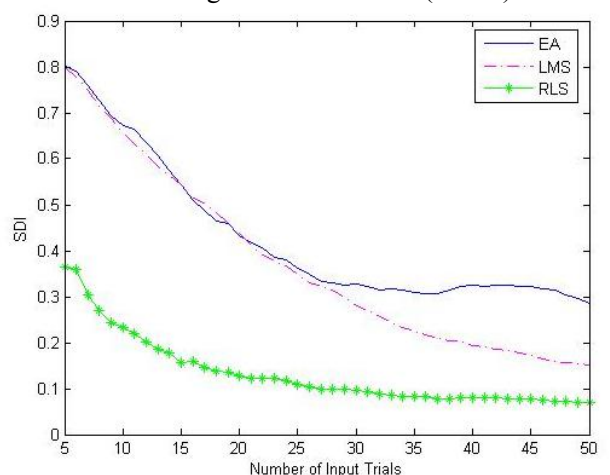

(b) $-15 \mathrm{~dB}$

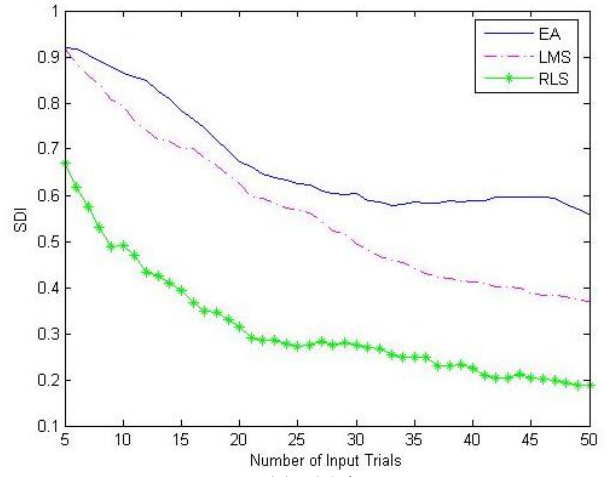

(c) $-20 \mathrm{~dB}$

Figure 5. Comparison of EA-SEP, LMS-SEP and RLS-SEP with template distortion index against trial number at different SNR 
TABLE 1. MEAN WAVEFORM CORRELATION COEFFICIENTS (MEAN \pm SD) FOR FIVE CASES BETWEEN (I) EA-SEP, (II) LMS-SEP, (III) RLS-SEP, AND THE

\begin{tabular}{cccc}
\multicolumn{4}{c}{ TEMPLATE } \\
\hline $\mathbf{- 1 0 d B}$ & $0.90 \pm 0.01$ & $0.90 \pm 0.01$ & $0.93 \pm 0.02$ \\
$\mathbf{- 1 5 d B}$ & $0.76 \pm 0.03$ & $0.78 \pm 0.03$ & $0.89 \pm 0.01$ \\
$\mathbf{- 2 0 d B}$ & $0.53 \pm 0.04$ & $0.58 \pm 0.05$ & $0.74 \pm 0.04$ \\
\hline
\end{tabular}

TABLE 2. MEAN PERCENTAGE DIFFERENCE (MEAN \pm S.D.) OF THE PEAK LATENCY AND THE AMPLITUDE (N1-P1) BETWEEN (I) EA-SEP , (II) LMS-SEP, (III) RLS-SEP, AND THE TEMPLATE AT DIFFERENT SNR

\begin{tabular}{cccc}
\hline SNR & Latency N1 & Latency P1 & Amplitude \\
\hline $\boldsymbol{E} \boldsymbol{A}-\boldsymbol{S E P}$ & & & \\
$\mathbf{- 1 0 d B}$ & $5.2 \pm 3.3$ & $1.2 \pm 1.5$ & $3.5 \pm 6.8$ \\
$\mathbf{- 1 5 d B}$ & $3.6 \pm 3.6$ & $4.4 \pm 6.0$ & $2.2 \pm 0.1$ \\
$\mathbf{- 2 0 d B}$ & $5.6 \pm 3.0$ & $3.3 \pm 2.1$ & $3.0 \pm 0.4$ \\
$\boldsymbol{L} \boldsymbol{M S}-\boldsymbol{S E P}$ & & & \\
$\mathbf{- 1 0 d B}$ & $5.2 \pm 3.3$ & $1.2 \pm 1.5$ & $3.3 \pm 5.6$ \\
$\mathbf{- 1 5 d B}$ & $3.8 \pm 3.4$ & $1.8 \pm 1.7$ & $2.1 \pm 0.1$ \\
$\mathbf{- 2 0 d B}$ & $4.9 \pm 3.1$ & $2.9 \pm 1.6$ & $2.8 \pm 0.4$ \\
$\boldsymbol{R} \boldsymbol{L S}-\boldsymbol{S E P}$ & & & \\
$\mathbf{- 1 0 d B}$ & $2.9 \pm 3.9$ & $1.7 \pm 1.2$ & $6.5 \pm 3.4$ \\
$\mathbf{- 1 5 d B}$ & $6.5 \pm 3.1$ & $0.3 \pm 0.4$ & $1.5 \pm 0.2$ \\
$\mathbf{- 2 0 d B}$ & $9.0 \pm 5.2$ & $3.3 \pm 3.0$ & $1.9 \pm 0.2$ \\
\hline
\end{tabular}

\section{DISCUSSION}

The objective of this paper is to compare the performance of three algorithms used for SEP extraction. Except for the EA, the other two are all used in ANC. As an ANC filter, the key point of the effectiveness lies in how to choose the reference channel. This requires the knowledge of the categories of noise, which arises from the human scalp. Practically, noises could be classified as the bio-inspired and system-related [5]. From the clinical experience, EEG is the most influential bio-inspired noise to the surface SEP recorded from conscious subjects, because EEG signals are generated continuously by the subject as long as the acquisition process lasts [9]. Continuous EEG recorded from A1-Fz was noted to contain less SEP signal [10]. The A1-Fz and the $\mathrm{Cz}-\mathrm{Fz}$ should be correlated with each other but not exactly the same since the waveform may change during the propagation [11]. Regarding those system related noises, most of them are considered to be white Gaussian. Therefore, in this simulation study, the background noise of SEP is the sum of EEG and WGN.

\section{CONCLUSION}

This paper proposed the RLS-ANC for SEP extraction. The performance of RLS-ANC was compared with the LMS-ANC in simulation and experimental study. The experimental results showed that the RLS-ANC outperforms than the LMS-ANC and EA for SEP extraction. It is confident to conclude that the RLS-ANC is able to provide valuable medical information for practical spinal cord function detection and monitoring in operating theaters.

\section{REFERENCES}

[1] Turner S., Picton P., and Campbell J., "Extraction of short-latency evoked potentials using a combination of wavelets and evolutionary algorithms," Medical Engineering \& Physics, 2003, 25:407-412.

[2] McGillem C. D., Aunon J. I., and Childers D. G., "Signal processing in evoked potential research: Applications of filtering and pattern recognition," Crit. Rev. Bioeng., CRC, Oct., 1981, vol. 9, pp. 225-265.

[3] MacLennan A. R. and Lovely D. F., "Reduction of evoked potential measurement time by a TMS320 based adaptive matched filter," Med. Eng. Phys., vol. 17, pp. 248-56, Jun. 1995.

[4] Woody C. D., "Characterization of an adaptive filter for the analysis of variable latency neuroelectric signals," Med. Biol. Eng., 1967, 5:539-53.

[5] B. S. Lam, Y. Hu, W. W. Lu, K. D. Luk, C. Q. Chang, W. Qiu, and F. H. Chan, "Multi-adaptive filtering technique for surface somatosensory evoked potentials processing," Med. Eng. Phys., Apr. 2005, vol. 27, pp. 257-66.

[6] Simon Haykin, "Adpative filter theory (4th edition)," Prentice Hall, September 24, 2001.

[7] Widrow B., Glover J. R., McCool J. M., Williams C. S., Hearn R. H., et al. "Adaptive noise canceling: principles and applications," Proc. IEEE 1975, 63(12): $1692-716$.

[8] Eweda E. and Macchi O., "Convergence of the RLS and LMS adaptive filters," 1EEE Trans. Circuits. Smt., vol. CAS-34, no. 7, pp. 799-803, July 1987.

[9] Gobbele R., Waberski T. D., Kuelkens S., Sturm W., Curio G., and Buchner H., "Thalamic and cortical high-frequency $(600 \mathrm{~Hz})$ somatosensory evoked potential (SEP) components are modulated by slight arousal changes in awake subjects," Exp. Brain Res., 2000, 133(4):506-13.

[10] Tinazzi M., Zanette G., Fiaschi A., andMauguiere F., "Effect of stimulus rate on the cortical posterior tibial nerve SEPs: a topographic study," Electroencephalogr Clin Neurophysiol, 1996, 100(3):210-9.

[11] Kaminski M., Blinowska K., and Szclenberger W., "Topographic analysis of coherence and propagation of EEG activity during sleep and wakefulness," Electroen-cephalogr Clin Neurophysiol 1997, 102(3): 216-27. 